\title{
Synchronization of Extended Chaotic Systems with Long-Range Interactions: An Analogy to Lévy-Flight Spreading of Epidemics
}

\author{
Claudio Juan Tessone, ${ }^{1,2}$ Massimo Cencini, ${ }^{3}$ and Alessandro Torcini ${ }^{2,4}$ \\ ${ }^{1}$ Institut Mediterrani d'Estudis Avançats, CSIC-UIB, Edifici Mateu Orfila, Campus UIB, 07122 Palma de Mallorca, Spain \\ ${ }^{2}$ Istituto dei Sistemi Complessi - CNR, via Madonna del Piano 10, 50019 Sesto Fiorentino, Italy \\ ${ }^{3}$ INFM-CNR, SMC Dipartimento di Fisica, Università di Roma "La Sapienza”, piazzale A. Moro 2, O0185 Roma, Italy \\ and Istituto dei Sistemi Complessi - CNR, via dei Taurini 19, 00185 Roma, Italy \\ ${ }^{4}$ INFN - Sezione di Firenze, via Sansone 1, 50019 Sesto Fiorentino, Italy
}

(Received 28 July 2006; published 29 November 2006)

\begin{abstract}
Spatially extended chaotic systems with power-law decaying interactions are considered. Two coupled replicas of such systems synchronize to a common spatiotemporal chaotic state above a certain coupling strength. The synchronization transition is studied as a nonequilibrium phase transition and its critical properties are analyzed at varying the interaction range. The transition is found to be always continuous, while the critical indexes vary with continuity with the power-law exponent characterizing the interaction. Strong numerical evidences indicate that the transition belongs to the anomalous directed percolation family of universality classes found for Lévy-flight spreading of epidemic processes.
\end{abstract}

PACS numbers: 05.45.Xt

Since its discovery [1], chaotic synchronization has become a very active and important field of research [2], especially for its applications in control and secure communications [3,4]. It has been observed in various experimental setups from semiconductor lasers to chemical reactions [5]. The synchronization transition (ST) is particularly interesting in spatially extended chaotic systems [6] where, due to the spatial dependence, a parallel with nonequilibrium phase transitions can be drawn. Extensive numerical studies of coupled map lattices (CML), which are prototype models for space-time chaos [7], have shown that ST between two replicas of CML with short-range interactions is continuous and the associated critical properties belong to two distinct universality classes depending on the nature of the individual elements of the system [811]. For smooth chaotic maps, linear mechanisms rule information propagation, and ST occurs as soon as the transverse Lyapunov exponent crosses zero. In this case, the transition belongs to the multiplicative noise universality class [12]. For discontinuous maps, a linear stability analysis is no more sufficient to locate the ST, and the replicas synchronize (for definitely negative transverse Lyapunov exponent) when the spreading velocity of finite amplitude perturbations vanishes [8]. The synchronization in such systems is controlled by nonlinear effects and the transition belongs to the directed percolation (DP) universality class [13], analogously to that found for cellular automata [14]. At present we thus have a satisfactory understanding of the critical properties of ST for shortrange coupled chaotic systems $[9,10]$.

The aim of this Letter is to study the synchronization transition of extended chaotic systems with long-range spatial coupling. Long-range interactions are indeed relevant to many real systems such as neuron populations [15], Josephson junctions [16], cardiac pacemaker cells [17], and to the issue of disease spread triggered by aviation traffic [18]. We consider, as a prototype of systems with

0031-9007/06/97(22)/224101(4)

224101-1 long-range interacting elements, a model of CMLs with coupling decaying as a power law [19-21]. As a first step we investigate the case of maps whose dynamics is dominated by nonlinear mechanisms. We show that the critical indexes characterizing the transition depend continuously upon the exponent $\sigma$, which sets the coupling range. Moreover, strong numerical evidences indicate that the critical exponents are compatible with those found in a model for anomalous directed percolation studied by Hinrichsen and Howard [22] (HH in the following).

Anomalous DP arises in epidemic spreading whenever the infective agent can perform unrestricted Lévy flights $[22,23]$. Such processes, originally introduced in Ref. [24], can be modeled by assuming, e.g., in $d=1$, that an already infected site propagates the disease to any other site with a probability $P(r) \sim r^{-(1+\sigma)}$ algebraically decaying with the distance $r$ (similarly to the distance distribution of human travels, which are nowadays responsible for the geographical spread of infectious diseases [18] ). Numerical studies of a stochastic lattice model, which generalizes directed bond percolation, have shown that the critical exponents vary continuously with $\sigma$ [22], despite the divergence of the average interaction distance for $\sigma<1$ and of the second moment of $P(r)$ for $\sigma<2$. These findings confirm previous theoretical results [23] according to which usual DP should be recovered for sufficiently shortrange coupling [namely, for $\sigma>\sigma_{c} \equiv 2.0677(2)$ ] and the mean-field description should become exact for $\sigma<$ $\sigma_{m} \equiv 0.5$. Furthermore, close to $\sigma_{m}$, the critical exponents found by $\mathrm{HH}$ agree with renormalization group calculations [23].

We investigate the synchronization transition of two coupled replicas of CML defined as:

$$
\begin{aligned}
& x_{i}(t+1)=(1-\gamma) F\left(\tilde{x}_{i}(t)\right)+\gamma F\left(\tilde{y}_{i}(t)\right) \\
& y_{i}(t+1)=(1-\gamma) F\left(\tilde{y}_{i}(t)\right)+\gamma F\left(\tilde{x}_{i}(t)\right),
\end{aligned}
$$


where $\tilde{z_{i}}=\left\{\tilde{x}_{i}, \tilde{y}_{i}\right\}$ is given by:

$$
\tilde{z_{i}}=(1-\epsilon) z_{i}+\frac{\epsilon}{\eta(\sigma)} \sum_{m=1}^{M} \frac{z_{i-j_{m}(q)}+z_{i+j_{m}(q)}}{\left[j_{m}(q)\right]^{\sigma}} .
$$

The labels $t$ and $i$ are the discrete temporal and spatial indexes, $x_{i}(t), y_{i}(t) \in[0,1]$ are the state variables, $L$ is the lattice size $(i=1, \ldots, L)$, and periodic boundary conditions are assumed [i.e., $\left.z_{i+L}(t)=z_{i}(t)\right]$. As local dynamics, throughout this work, we employ the Bernoulli map $F(x)=2 x(\bmod 1)$, which is characterized by a discontinuity that makes the nonlinear effects dominating [25]. The interaction range is controlled by the exponent $\sigma$ : for $\sigma=$ 0 globally coupled maps are obtained, while standard CMLs with nearest neighbor coupling are recovered in the limit $\sigma \rightarrow \infty$. Two coupling constants $\epsilon$ and $\gamma$ enter the system definition. The former measures the amplitude of the spatial coupling and is fixed to $2 / 3$. The latter controls the strength of the sitewise interaction between the two replicas. In the range of $\sigma$ examined, a synchronized state is always achieved as the coupling exceeds a certain $\gamma_{c}=\gamma_{c}(q, \sigma)$.

Usually the model has been studied in the fully coupled case [19-21], i.e., for $j_{m}(q) \equiv m$ with $M=(L-1) / 2$. Instead, by following Ref. [26], we consider here a modified version of the coupling, where $j_{m}(q)=q^{m}-1$ with $q$ values typically chosen as $q=2,4$, and 8 . The sum in Eq. (2) extends to $M=\log _{q}(L / 2)$; finally $\eta(\sigma)=$ $2 \sum_{m=1, M}\left[j_{m}(q)\right]^{-\sigma}$ is a normalization factor. The rationale for the choice $j_{m}(q)=q^{m}-1$ lies in its convenience from a computational point of view. As a matter of fact, the chosen coupling scheme allows for simulating very large systems: each updating step can be performed in $\mathcal{O}\left(L \log _{q} L\right)$ operations instead of $\mathcal{O}\left(L^{2}\right)$ needed in the fully coupled case. Moreover, a simple reasoning shows that the model with $j_{m}(q)=q^{m}-1$ and exponent $\sigma$ exhibits the same critical properties of the fully coupled one with a different exponent $\sigma_{f c}=\sigma+1$. Indeed, both versions of the model should display the same critical properties once the spatial interactions scale analogously. For the modified model the coupling weight over the interval $\left[j_{m}(q), j_{m+1}(q)\right]$ containing a single coupled site is simply $1 / j_{m+1}(q)^{\sigma} \sim q^{-(m+1) \sigma}$, while for the fully coupled case this amounts to $\sum_{k=j_{m}(q)+1}^{j_{m+1}(q)} k^{-\sigma_{f c}} \sim q^{-(m+1)\left(\sigma_{f c}-1\right)}$. Therefore, the two weights scale in the same manner only for $\sigma_{f c}=\sigma+1$ as confirmed by numerical results reported in the following.

To characterize the ST it is useful to introduce the synchronization error $w_{i}(t)=\left|x_{i}(t)-y_{i}(t)\right|$. Its spatiotemporal evolution is reported in Fig. 1 for various values of $\sigma$ just above the synchronization transition $\left(\gamma \geq \gamma_{c}\right)$. In the short-range limit $\sigma \rightarrow \infty$, percolating structures, typical of ordinary DP, are clearly observable. As the range of the interaction increases (i.e., $\sigma$ decreases) the spatial structures tend to be smoothed out. Finally, for $\sigma \lesssim 0.5$, the spatial structures are no more detectable.

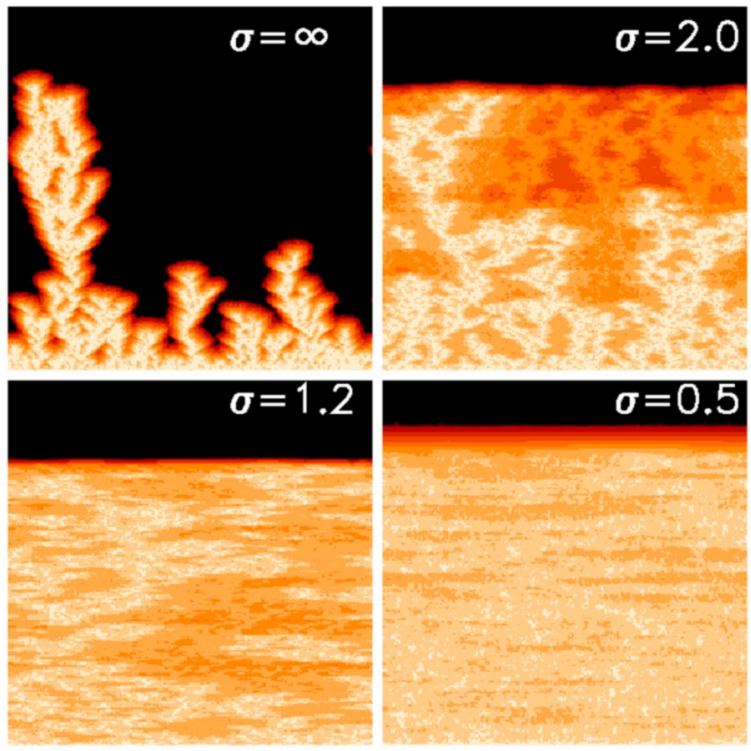

FIG. 1 (color online). Space-time plot of synchronization error $w_{i}(t)=\left|x_{i}(t)-y_{i}(t)\right|$ for two replicas of the CML (1-2) for $q=$ 2 and $M=9$ with $\gamma$ slightly larger than $\gamma_{c}$ and different $\sigma$ 's, see labels. The space (respective time) axis correspond to the horizontal (respective vertical) axis. The colors code the absolute value of the synchronization error in logarithmic scale. Colors go from intense white (yellow online) corresponding to $w_{i}(t) \sim 1$ to dark black associated to the fully synchronized state, i.e., $w_{i}(t)=0$.

To be more quantitative, we consider the spatial average of the synchronization error $\rho_{\gamma}(t)=\sum_{i} w_{i}(t) / L$ which is the natural order parameter: it vanishes, at sufficiently long times, whenever a complete synchronization is achieved, i.e., $\rho_{\gamma}^{*}=\lim _{t \rightarrow \infty} \rho_{\gamma}(t)=0$ for $\gamma>\gamma_{c}$; while it remains finite at any time in the unsynchronized state, i.e., for $\gamma<$ $\gamma_{c}$. In terms of $\rho_{\gamma}(t)$, we can now define the critical exponents $\delta, \beta$, and $z$ that will be function of $\sigma: \delta$ rules the temporal scaling of the order parameter at the critical point $\gamma=\gamma_{c}$, i.e., $\rho_{\gamma_{c}}(t) \sim t^{-\delta} ; \beta$ controls the way the asymptotic value of the order parameter approaches zero, $\rho_{\gamma}^{*} \sim\left(\gamma_{c}-\gamma\right)^{\beta}$ for $\gamma \leq \gamma_{c}$; and finally the dynamical exponent $z$ can be defined in terms of the finite-size scaling relation, valid at the critical point:

$$
\rho_{\gamma_{c}}(t) \sim L^{-\delta z} f\left(t / L^{z}\right)
$$

These exponents, which fully characterize the transition (ruling the divergence of, e.g., the spatial and temporal correlation lengths at the critical point), have been measured for different values of $\sigma$, by means of the following procedure [see Fig. 2(a)-2(c) for an illustration of the method and to appreciate the quality of the results]. The critical coupling $\gamma_{c}$ is estimated by examining the temporal behavior of $\rho_{\gamma}(t)$, averaged over many random initial conditions, for different values of $\gamma$. In particular, $\gamma_{c}$ is determined (typically with an absolute error within the range $\left.\left[2 \times 10^{-5}, 2 \times 10^{-4}\right]\right)$ as the point at which a scaling 

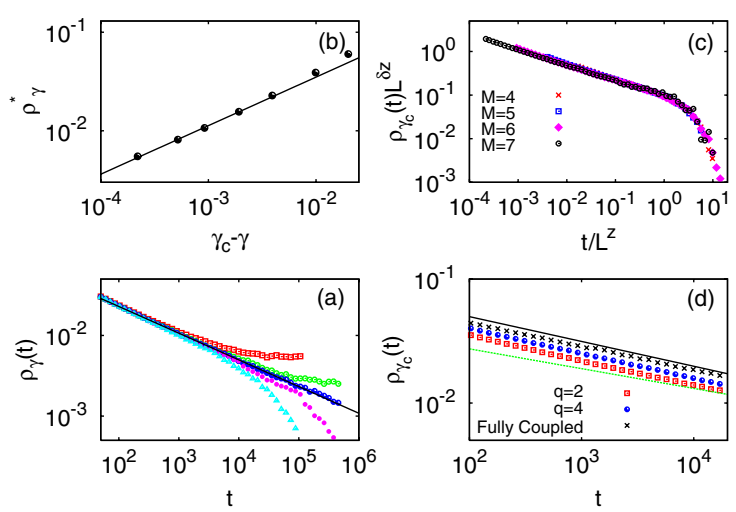

FIG. 2 (color online). (a) Average synchronization error $\rho_{\gamma}$ vs time for the CML (1) with $q=4$ and $M=8$ for $\sigma=1.4$; the curves refer to $\gamma=0.2977,0.29789,0.29792,0.29797,0.2981$ (from top to bottom). Here $\gamma_{c}=0.29792(2)$. The straight line indicates the slope $\delta=0.33(2)$ as obtained by a best fit. (b) Asymptotic synchronization error $\rho_{\gamma}^{*}$ vs $\gamma_{c}-\gamma$ for the same parameters as in (a); the straight line indicates the slope $\beta=0.49(5)$. (c) Finite-size scaling $\rho_{\gamma_{c}}(t) L^{\delta z}$ vs $t / L^{z}$ from which we estimate $z=1.05(5)$; symbols refer to different sizes $L=2 q^{M}$ (here $q=4$ ) and $M$ as in the legend. (d) The synchronization error $\rho_{\gamma_{c}}$ vs time at criticality for $\sigma=2$ using $q=2$ with $M=16$ and $q=4$ with $M=8$ together with the results for the fully coupled model for $\sigma_{f c}=1+\sigma=3$ and size $L=8$, 193. The top straight line displays the slope $\delta=0.2$ as estimated by a best fit; the bottom straight line shows the (short-range) DP value $\delta=0.15945$. Data are obtained by averaging $\rho_{\gamma}(t)$ over many $(\sim 100)$ different initial conditions.

behavior for $\rho_{\gamma}(t)$ emerges [see Fig. 2(a)]. With this method $\delta$ is directly computed by means of a best fit procedure on $\rho_{\gamma_{c}}(t)$. Once $\gamma_{c}$ is known, $\beta$ is obtained by measuring the saturation values $\rho_{\gamma}^{*}$ in the desynchronized regime (i.e., for $\gamma<\gamma_{c}$ ) for sufficiently long chains; see Fig. 2(b). Finally, performing several simulations with different sizes at the critical point $\gamma=\gamma_{c}$, the exponent $z$ is estimated through a data collapse based on Eq. (3). Although this procedure works satisfactorily [Fig. 2(c)] it is affected by large errors due to the unavoidable subjectivity in judging the collapse.

We remark that, as typical of strongly nonlinear maps [8,21], $\gamma_{c}$ cannot be identified by the condition of vanishing transverse Lyapunov exponent and, moreover, it depends not only on $\sigma$ but also on $q$. However, the critical exponents, apart from finite-size effects, are not influenced by $q$ as one can judge from Fig. 2(d), where the synchronization error at the critical point is shown for $q=2,4$ and $\sigma=2$. In the figure we also display the result for the fully coupled model with the exponent ruling the interaction range set to $\sigma_{f c}=3$. The data confirm that $\sigma_{f c}=1+\sigma$ as previously discussed.

In Fig. 3, we display the exponents $\beta$ and $\delta$ as a function of $\sigma$, and compare them with the results of $\mathrm{HH}$ obtained for stochastic models of Lèvy-flight epidemic spreading [22]. The agreement between the two sets of data is rather good.

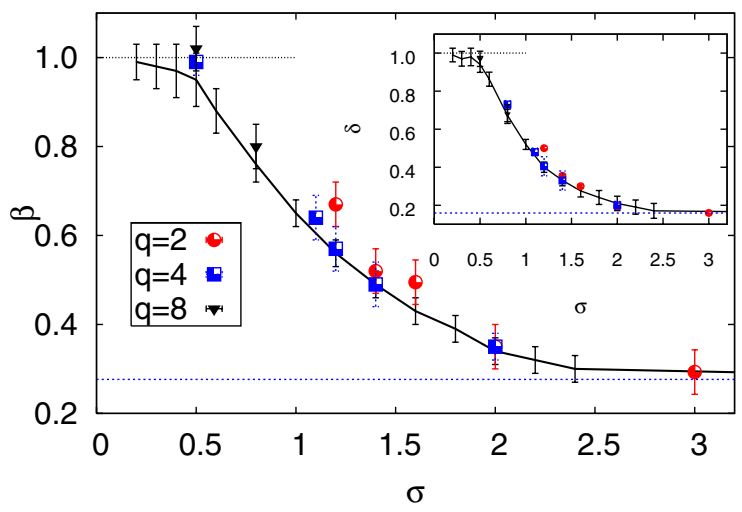

FIG. 3 (color online). Critical exponent $\beta$ vs $\sigma$ obtained from best fits using different lengths and basis $q=2,4,8$. The black thick curve represents the data of $\mathrm{HH}$ [22], the bottom straight line is the DP value, while the top one represents the mean-field prediction by $\mathrm{HH}$. CML with sizes from $L=2^{16} \approx 6 \times 10^{4}$ up to $L=2 \times 8^{7} \approx 4 \times 10^{6}$ maps have been used. Inset: critical exponent $\delta$ vs $\sigma$ symbols are as in the main panel.

Moreover, by increasing $q$ and so the maximal affordable spatial length $L$, we observe that the exponents approach those of $\mathrm{HH}$. This leads us to conclude that discrepancies are mainly due to finite-size effects. The results for $z$ (not reported) also confirm the overall agreement. Because of the strong finite-size effects and to computational limitations it was not possible to analyze systems with $\sigma<0.5$. For anomalous DP, it has been shown that in the interaction range $\left[\sigma_{m}, \sigma_{c}\right]$ the following scaling relation holds $[22,23]$

$$
\Delta=1-\sigma+(1-2 \delta) z \equiv 0 .
$$

As shown in Table I the relation (4) is fulfilled within the errors, strengthening the parallel between ST in spatiotemporal chaotic systems with power-law interactions and anomalous DP. It is worth stressing that it is absolutely nontrivial that the deterministic system here investigated was in quantitative agreement with the stochastic one studied in $\mathrm{HH}$. The fact that the critical exponents are the same suggests that the universality class (actually the family of universality classes labeled by $\sigma$ ) is the same for both models.

The agreement of the critical exponents with those of Ref. [22] (shown in Fig. 3) represents a strong indication that the synchronization dynamics associated to model (1) and (2) should admit the same stochastic field description proposed for anomalous directed percolation [22,23]:

$$
\partial_{t} n=D_{N} \nabla^{2} n+D_{A} \nabla^{\sigma} n+\tau n-\lambda n^{2}+g(n) \xi,
$$

where, in the present context, $n$ represents the coarse grained synchronization error obtained by averaging $w_{i}(t)$ over a suitable space-time cell. The parameters entering Eq. (5) are the standard and anomalous diffusion coefficients $D_{N}$ and $D_{A}$, respectively, $\tau$ that measures the distance from the critical point (i.e., from the synchronization threshold $\gamma_{c}$ ) and the amplitude of the nonlinear 
TABLE I. Scaling relation (4) for various values of $\sigma$, for each measurement the corresponding basis $q$ is reported. The measured $\Delta$ are compatible with zero in the range $\left[\sigma_{m}, \sigma_{c}\right]$. See text for details.

\begin{tabular}{lcccccr}
\hline \hline$\sigma[q]$ & $3.0[2]$ & $2.0[4]$ & $1.4[4]$ & $1.2[4]$ & $0.8[8]$ & $0.5[8]$ \\
\hline$\Delta$ & $0.93(6)$ & $-0.13(9)$ & $-0.04(12)$ & $-0.03(10)$ & $-0.03(23)$ & $0.04(13)$ \\
\hline \hline
\end{tabular}

term $\lambda$. The fractional derivative $\nabla^{\sigma}$ is nonlocal and can be defined through its action in Fourier space $\nabla^{\sigma} \rightarrow-|k|^{\sigma}$. Finally, $\xi$ is a zero-average $\delta$-correlated (in space and time) Gaussian noise field with unit variance and $g^{2}(n) \propto$ $n$. For systems with short-range interactions, Eq. (5) with $D_{A}=0$ (i.e., the field description of ordinary DP) was heuristically derived in [11] from a simple stochastic model, mimicking the behavior of $w_{i}(t)$ for Bernoulli maps. That derivation can be straightforwardly extended to the present case giving Eq. (5) as a macroscopic description of the dynamics. Indeed, the only difference comes from the spatial coupling that can be handled as in [27] (where the synchronization of long-range coupled chaotic oscillators was considered) leading to the fractional derivative term. However, a detailed derivation goes beyond the scopes of the present Letter.

In conclusion, we have provided strong numerical evidence that the synchronization transition of two coupled replicas of a chain of discontinuous maps with long-range interactions is characterized by a continuum of universality classes labeled by the exponent $\sigma$, which sets the interaction range. Moreover, the critical exponents agree with those reported for anomalous directed percolation [22]. Preliminary results indicate that also for continuous maps the critical exponents depends on $\sigma$ and that they do not belong to the anomalous DP universality class. By analogy with the present results, we conjecture that the critical properties of the synchronization transition for continuous maps should be reproducible in terms of a multiplicative noise Langevin equation with long-range interactions [i.e., an equation similar to (5) but with $g(n) \propto n$ [12]]. To the best of our knowledge this equation has not been yet subject of theoretical or numerical analysis. It will be definitely worth the task to undertake such a study in the future.

We are grateful to F. Cecconi and F. Ginelli for useful discussions, remarks, and for a careful reading of the manuscript. M. C. acknowledges partial support from the PRIN Grant for 2005 "Statistical Mechanics of Complex Systems" by MIUR and MPIPKS for computational resources.

[1] H. Fujisaka, Prog. Theor. Phys. 70, 1264 (1983); H. Fujisaka and T. Yamada, Prog. Theor. Phys. 69, 32 (1983).

[2] A. Pikovsky, M. Rosenblum, and J. Kurths, Synchronization A Universal Concept in Nonlinear Sciences (Cambridge University Press, Cambridge, England, 2001).
[3] L. M. Pecora and T. L. Carroll, Phys. Rev. Lett. 64, 821 (1990); Phys. Rev. A 44, 2374 (1991).

[4] G. D. Van Wiggeren and R. Roy, Science 279, 1198 (1998); A. Argyris et al., Nature (London) 437, 343 (2005).

[5] W. Wang, I. Z. Kiss, and J. L. Hudson, Chaos 10, 248 (2000); D. J. DeShazer, R. Breban, E. Ott, and R. Roy, Phys. Rev. Lett. 87, 044101 (2001).

[6] M. C. Cross and P. H. Hohenberg, Rev. Mod. Phys. 65, 851 (1993).

[7] K. Kaneko, Prog. Theor. Phys. 72, 480 (1984).

[8] L. Baroni, R. Livi, and A. Torcini, Phys. Rev. E 63, 036226 (2001); F. Bagnoli and F. Cecconi, Phys. Lett. A 282, 9 (2001).

[9] V. Ahlers and A. Pikovsky, Phys. Rev. Lett. 88, 254101 (2002).

[10] M. A. Muñoz and R. Pastor-Satorras, Phys. Rev. Lett. 90, 204101 (2003).

[11] F. Ginelli, R. Livi, A. Politi, and A. Torcini, Phys. Rev. E 67, 046217 (2003).

[12] M.A. Muñoz, in Advances in Condensed Matter and Statistical Mechanics, edited by E. Korutcheva et al. (Nova Science Publishers, New York, 2004).

[13] H. Hinrichsen, Adv. Phys. 49, 815 (2000).

[14] P. Grassberger, Phys. Rev. E 59, R2520 (1999).

[15] M. Dhamala, V. K. Jirsa, and M. Ding, Phys. Rev. Lett. 92, 028101 (2004).

[16] K. Wiesenfeld, P. Colet, and S.H. Strogatz, Phys. Rev. Lett. 76, 404 (1996).

[17] C. Peskin, Mathematical Aspects of Heart Physiology (New York University, New York, 1975).

[18] L. Hufnagel, D. Brockmann, and T. Geisel, Proc. Natl. Acad. Sci. U.S.A. 101, 15124 (2004); D. Brockmann, L. Hufnagel, and T. Geisel, Nature (London) 439, 462 (2006).

[19] G. Paladin and A. Vulpiani, J. Phys. A 27, 4911 (1994); A. Torcini and S. Lepri, Phys. Rev. E 55, R3805 (1997).

[20] C. Anteneodo, S. E. de S. Pinto, A. M. Batista, and R. L. Viana, Phys. Rev. E 68, 045202(R) (2003).

[21] M. Cencini and A. Torcini, Physica (Amsterdam) D208, 191 (2005).

[22] H. Hinrichsen and M. Howard, Eur. Phys. J. B 7, 635 (1999).

[23] H. K. Janssen, K. Oerding, F. van Wijland, and H.J. Hilhorst, Eur. Phys. J. B 7, 137 (1999).

[24] D. Mollison, J. R. Stat. Soc. B 39, 283 (1977); P. Grassberger, in Fractals in Physics, edited by L. Pietronero and E. Tosatti (Elsevier, New York, 1986).

[25] The results here presented are expected to extend to continuous maps with sufficiently large first derivatives; see Refs. [8,9,11,21] for a detailed discussion of this issue.

[26] A. Torcini, Lect. Notes Phys. 457, 537 (1995).

[27] V.E. Tarasov and G. M. Zaslavsky, Chaos 16, 023110 (2006). 